\title{
Bubble size prediction in gas-solid fluidized beds using genetic programming
}

\author{
R. R. Sonolikar ${ }^{1}$, M. P. Patil ${ }^{1}$, R. B. Mankar ${ }^{2}$, S. S. Tambe ${ }^{3, *}$ and B. D. Kulkarni ${ }^{3}$ \\ ${ }^{1}$ Solid and Hazardous Waste Management Division, CSIR-National Environmental Engineering Research Institute, Nehru Marg, \\ Nagpur 440 020, India \\ ${ }^{2}$ Department of Chemical Engineering, Laxminarayan Institute of Technology, Amravati Road, Nagpur 440033 , India \\ ${ }^{3}$ Chemical Engineering and Process Development (CEPD) Division, CSIR-National Chemical Laboratory, Dr Homi Bhabha Road, \\ Pune 411 008, India
}

The hydrodynamics of a gas-solid fluidized bed (FB) is affected by the bubble diameter, which in turn strongly influences the performance of a fluidized bed reactor (FBR). Thus, determining the bubble diameter accurately is of crucial importance in the design and operation of an FBR. Various equations are available for calculating the bubble diameter in an FBR. It has been found in this study that these models show a large variation while predicting the experimentally measured bubble diameters. Accordingly, the present study proposes a new equation for computing the bubble diameter in a fluidized bed. This equation has been developed using an efficient, yet infrequently employed computational intelligence (CI)-based datadriven modelling method termed genetic programming (GP). The prediction and generalization performance of the GP-based equation has been compared with that of a number of currently available equations for computing the bubble diameter in a fluidized bed and the results obtained show a good performance by the newly developed equation.

Keywords: Bubble diameter, bubble motion, fluidized bed, genetic programming.

FLUIDIZED bed reactors (FBRs) are widely used in petroleum, chemical, food, metallurgical, pharmaceutical and power generation industries ${ }^{1}$. The design of gas-solid fluidized reactors requires an understanding of the size and behaviour of bubbles therein. Despite widespread use of FBRs, their scale-up still depends on the empirical methods owing to the complicated nature of gas-solid flows inside the reactor. The formation and travel of bubbles play a crucial role in the hydrodynamic study of a fluidized bed. Specifically, the size of a bubble decides the homogeneity, heterogeneity and slug formation in a fluidized bed. There are four stages of bubble motion: formation of bubble, its detachment from the orifice, travel in the bed and finally bursting. Harrison and Leung ${ }^{2}$, and Zenz $z^{3}$ proposed equations for the formation of a bubble at the orifice. Bubble detachment time was studied ${ }^{3-6}$ to

\footnotetext{
*For correspondence. (e-mail: ss.tambe@ncl.res.in)
}

propose equations for its prediction. A number of equations have also been proposed for determining the bubble diameter while the bubble is in motion, as a function of the particle diameter and density, bed geometry, type of distributor and gas velocity.

\section{Previous work}

Major studies concerning the bubble travel are described here. A number of studies have proposed equations to model the bubble dynamics ${ }^{7-21}$. As suggested by Patil et $a{ }^{22}$, the Darton's model is based on the bubbles tending to rise in preferred paths and that the distance travelled by the two neighbouring bubbles before coalescence is proportional to their lateral separation. Farshi et al. ${ }^{23} \mathrm{stu}-$ died and compared the performance of a number of equations with the experimental data and found that the equation by Rowe $^{15}$ fitted their experimental data the best. In the pilot scale study, the corresponding experimental data were well predicted by the equations of Rowe $^{15}$ and Darton et al. ${ }^{8}$. In a gas-solid bubbling fluidized bed, Farshi et al. ${ }^{23}$ suggested that the equations by Mori and $\mathrm{Wen}^{16}$ and Rowe ${ }^{15}$ possess a good bubble diameter predicting ability. Hilligard and Werther ${ }^{7}$ performed experiments using quartz sand particles belonging to the Geldart's Group B of $480 \mu \mathrm{m}$ size and density equal to $2640 \mathrm{~kg} / \mathrm{m}^{3}$. On the basis of these experiments, they proposed a relation for calculating the bubble diameter of the Geldart Group D particles.

The two commonly used variables in all the equations for calculating the bubble diameter are, the ratio of injection velocity $(U)$ to minimum fluidization velocity $\left(U_{\mathrm{mf}}\right)$ and the bed height $(h)$ of the bubble above the distributor (Table 1). The equations proposed ${ }^{11,17}$ show that the diameter of the bubble $\left(D_{\mathrm{b}}\right)$ is proportional to the particle diameter $\left(d_{\mathrm{p}}\right)$, whereas Park et al. ${ }^{13}$ reported $D_{\mathrm{b}}$ to be proportional to $\left(d_{\mathrm{p}}^{1.5}\right)$. Similarly, $D_{\mathrm{b}}$ is shown to be dependent on the density of the material ${ }^{11,17}$.

The present study focuses on the behaviour of the bubble diameter since the hydrodynamics of a bubbling fluidized bed strongly depends on the bubble characteristics. Owing to the variations found in the predictions of 
Table 1. Bubble diameter equations proposed by different authors

\begin{tabular}{|c|c|}
\hline Reference & Equation \\
\hline Yasui et al. ${ }^{11}$ & $D_{\mathrm{b}}=0.16 \rho_{\mathrm{p}} d_{\mathrm{p}}\left(\frac{U}{U_{\mathrm{mf}}}-1\right)^{0.63} h$ \\
\hline Whitehead and Young ${ }^{12}$ & $D_{\mathrm{b}}=9.76\left(\frac{U}{U_{\mathrm{mf}}}\right)^{0.33}(0.32 h)^{0.54}$ \\
\hline Park et al. ${ }^{13}$ & $D_{\mathrm{b}}=33.3 * 10^{3} d_{\mathrm{p}}^{1.5}\left(\frac{U}{U_{\mathrm{mf}}}-1\right)^{0.77} h$ \\
\hline Rowe et al. ${ }^{15}$ & $\begin{array}{l}D_{\mathrm{b}}=\frac{\left(U-U_{\mathrm{mf}}\right)^{0.5}\left(h+h_{0}\right)^{0.75}}{g^{0.5}} \\
\text { where } h=0 \text { for porous plate and for perforated plate; } h_{0}=0.772+0.006 h \\
D_{\mathrm{b}}=0.00853\left[1+27.2\left(U-U_{\mathrm{mf}}\right)^{0.4}\right]^{0.333}[1+6.84 h]^{1.21}\end{array}$ \\
\hline Darton et $_{\text {al. }}{ }^{8}$ & $D_{\mathrm{b}}=0.54\left[U-U_{\mathrm{mf}}\right]^{0.4}\left[h+4 \sqrt{A_{0}}\right]^{0.8} g^{-0.2}$ \\
\hline Hilligardt and Werther ${ }^{7}$ & $\begin{array}{l}D_{\mathrm{b}}=D_{0}\left(1+27\left(U_{0}-U_{\mathrm{mf}}\right)\right)^{1 / 3}(1+6.4 h)^{1.2} \\
h=\text { height above the distributor, } D_{0}=0.0061,0.0085 \text { and } 0.0123 \text { for Geldart group } \\
A, B \text { and } D \text { particles respectively }\end{array}$ \\
\hline Lim et al. ${ }^{10}$ & $\begin{array}{l}D_{\mathrm{b}}=\left(\frac{8\left(U_{0}-U_{\mathrm{mf}}\right)\left(2^{2} / 5-1\right)}{\pi \lambda g^{1 / 3}}+d_{0}^{3 / 2}\right)^{2 / 3} \\
D_{0}=\left[8\left(U_{0}-U_{\mathrm{mf}}\right) A_{0} / \pi \lambda g^{0.5}\right]^{2 / 3} \\
A_{0}=5.6 \times 10^{-5} \mathrm{~m}^{2} \text { for porous plate, } \lambda=2 \text { (dimensionless) }\end{array}$ \\
\hline
\end{tabular}

existing equations, a new equation has been proposed for calculating the bubble diameter accurately. This equation uses a computational intelligence (CI)-based exclusively data-driven modelling formalism known as genetic programming (GP), which possesses some novel and attractive features. Since the GP-based equation makes accurate predictions of bubble diameter, it is expected to be significantly useful for studying and scaling-up of the commercial fluidized bed reactors.

\section{Experimental set-up}

A two-dimensional gas-solid fluidized bed with a central jet (Figure 1) was constructed for studying the formation of a bubble and its travel in the bed. This bed has dimensions of $18 \mathrm{~cm} \quad$ (width) $\times 1.3 \mathrm{~cm} \quad$ (depth) $\times 100 \mathrm{~cm}$ (height). The jet at the centre has dimensions of $1 \mathrm{~cm} \times$ $1 \mathrm{~cm}$. A porous plate was used for the distribution of the gas and it generated a pressure drop equal to $15 \%$ of the total pressure drop of the fluidized bed. A plenum of dimensions $20 \mathrm{~cm} \quad$ (width) $\times 3 \mathrm{~cm} \quad($ depth $) \times 30 \mathrm{~cm}$ (height) was packed up to the height of $15 \mathrm{~cm}$ for maintaining a uniform distribution of the gas. Air was used for effecting the fluidization, whose flow rate was accurately measured through a set of four rotameters covering a range of 0-200 litre/min.

In this study, spherical solid glass particles of diameters 275, 390, 462 and 1380 microns were used. The minimum fluidization velocity $\left(U_{\mathrm{mf}}\right)$ in respect of these particles was found by increasing the velocity of the gas passing through the packed bed in small steps and recording the corresponding pressure drop $(\Delta P)$, as well as the bed height. The weight of the material loaded initially was noted. Plots were generated using the $\Delta P$ and its variation with the gas velocity for fluidizing as well as defluidizing the material. The point of intersection of two lines - the horizontal one for the bubbling fluidized bed and the decreasing one pertaining to the defluidization, was considered as the minimum fluidization velocity. The bed height noted at different gas velocities assisted in determining the bed voidage.

The bubble formation and its travel during the fluidization of glass particles were noted with a high speed five mega pixel digital video camera LG KU 990. This camera was placed at a distance of $50 \mathrm{~cm}$ from the column that was operated at the speed of 120 frames/sec with a field of vision of $20 \times 20 \mathrm{~cm}$. The ratio of the gas velocities [(injection velocity, $\left.U_{\mathrm{j}}\right) /($ minimum fluidization velocity, $\left.U_{\mathrm{mf}}\right)$ ] equal to 15 was used at the jet inlet. A total of five experimental runs were conducted for studying the behaviour of the particles of a specific size and each run was recorded for $5 \mathrm{~min}$. The fluidization behaviour of particles with different diameters, namely 275, 390, 462 and 1380 microns, was examined. Here, the average bubble diameter was calculated using diameters of 12 bubbles. In this calculation, bubbles showing coalescence or splitting were ignored. This averaging procedure for calculating the bubble diameter was employed at different heights in the fluidized bed. An open source image analysis software termed Image $\mathbf{J}$ was used for measuring the bubble diameter. The solid volume fraction was related to the light intensity using a grey scale wherein the maximum bright area (pixel) corresponds to the $100 \%$ void (gas), whereas the darkest area (pixel) signifies a complete solid 

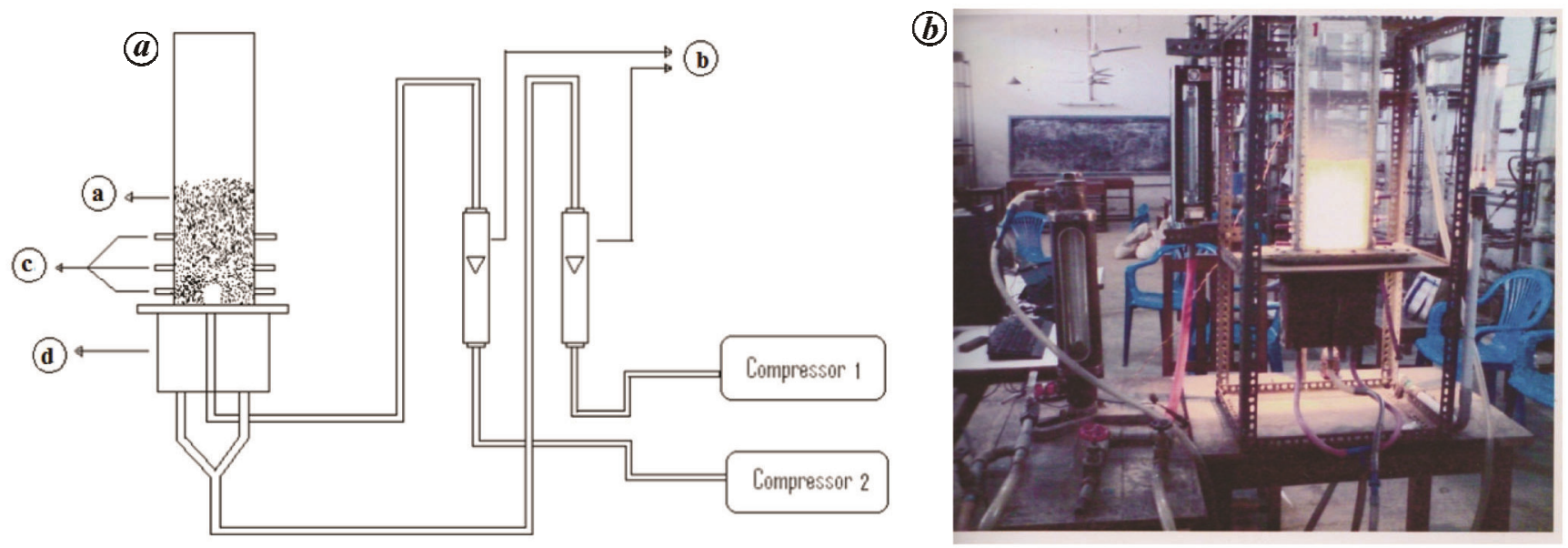

Figure 1. $\boldsymbol{a}$, Sketch of the experimental set-up: (a) fluidized bed, (b) rotameters, (c) pressure tapings, (d) plenum. $\boldsymbol{b}$, Photograph of the experimental set-up.

in the recorded image. For measuring the bubble diameter, $80 \%$ of the dark interface around the bubble was kept as the cut-off line. These data supplemented with the experimental measurements from other studies ${ }^{8,9,15,17}$ are listed in Table 2 and were used in developing the GPbased equation for predicting the bubble diameter.

\section{Modelling method}

Genetic programming (GP) is a computational intelligence $(\mathrm{CI})$-based exclusively data-driven modelling paradigm. It was proposed by $\mathrm{Koza}^{24}$ to automatically generate computer programs performing pre-defined tasks. Another important application of GP is known as 'symbolic regression (SR)', which is relevant to the present study. Given an example set consisting of the independent and dependent variables, the GP-based symbolic regression (hereafter termed 'GPSR') searches and optimizes the form of an appropriate linear or a nonlinear data fitting function and all the parameters associated with it. For performing the symbolic regression, GP uses the 'survival of the fittest' and 'genetic propagation of characteristics' principles of the Darwinian evolution followed by the biologically evolving species. Specifically, GP first generates an initial random population of candidate (probable) solutions (mathematical expressions/models) to the symbolic regression problem at hand. All the candidate expressions are coded using a tree-like structure with its branches formed by the randomly chosen function and terminal nodes. The function nodes represent mathematical operations, such as addition, subtraction, multiplication, division, exponentiation and logarithm, whereas the independent variables and parameters of the data fitting function are denoted by terminal nodes. The sizes of the trees encoding candidate expressions in symbolic forms can differ to enable forma- tion of equations of varying complexity and lengths. Given an example data set consisting of model inputs and output, a typical GPSR run implements the following steps.

(i) Initialization: Using tree structures, a random initial population of candidate solutions is created for a given data-fitting problem.

(ii) Fitness evaluation and selection: The fitter solutions are selected from the current candidate population to undergo crossover; the fitness is assessed using a fitness function that assigns a value (score) to a candidate solution depending upon how well it fulfils the task of data-fitting. A higher (lower) fitness value is assigned to a candidate solution that predicts the outputs with better (lower) accuracy. Having computed the fitness values of all candidates in the current population, a 'parent' pool is formed of solutions possessing relatively high fitness values. Methods such as roulette wheel selection and tournament selection are used for creating the stated pool.

(iii) Crossover: This is the most important GPSR step that forms a new generation of candidate solutions (offspring) using the candidates from the parent pool. Specifically, a pair of candidate solutions is selected randomly from the parent pool, and stochastically chosen parts of each parent tree are exchanged mutually between them and recombined to create two offspring solution trees. This step is repeated till a population of the desired number of offspring is formed.

(iv) Mutation: Here, a small change is randomly incorporated into the offspring candidate solutions generated in the crossover step. Specifically, a function (terminal) node in the offspring tree is replacedusing a small probability - with another function (terminal) node. 
RESEARCH ARTICLES

Table 2. Experimental data used in the construction of the GP-based drag model

\begin{tabular}{|c|c|c|c|c|c|c|}
\hline$D_{\mathrm{b}}(\mathrm{cm})$ & $h(\mathrm{~cm})$ & $d_{\mathrm{p}}(\mathrm{cm})$ & $U_{\mathrm{mf}}(\mathrm{cm} / \mathrm{s})$ & $U(\mathrm{~cm} / \mathrm{s})$ & $\rho\left(\mathrm{g} / \mathrm{cm}^{3}\right)$ & Authors \\
\hline 1.40 & 1.76 & 0.0692 & 33.8 & 67.6 & 2.545 & Patil et al. ${ }^{22}$; Material used: glass spheres $\left(\rho=2.545 \mathrm{~g} / \mathrm{cm}^{3}\right)$ \\
\hline 2.19 & 4.16 & 0.0692 & 33.8 & 67.6 & 2.545 & \\
\hline 2.80 & 3.77 & 0.0692 & 33.8 & 67.6 & 2.545 & \\
\hline 3.59 & 3.97 & 0.0692 & 33.8 & 67.6 & 2.545 & \\
\hline 2.80 & 5.98 & 0.0692 & 33.8 & 67.6 & 2.545 & \\
\hline 2.36 & 6.96 & 0.0692 & 33.8 & 67.6 & 2.545 & \\
\hline 3.31 & 6.89 & 0.0692 & 33.8 & 67.6 & 2.545 & \\
\hline 2.80 & 9.23 & 0.0692 & 33.8 & 67.6 & 2.545 & \\
\hline 3.20 & 9.23 & 0.0692 & 33.8 & 67.6 & 2.545 & \\
\hline 4.15 & 8.19 & 0.0692 & 33.8 & 67.6 & 2.545 & \\
\hline 2.86 & 12.94 & 0.0692 & 33.8 & 67.6 & 2.545 & \\
\hline 4.93 & 11.05 & 0.0692 & 33.8 & 67.6 & 2.545 & \\
\hline 8.30 & 11.05 & 0.0692 & 33.8 & 67.6 & 2.545 & \\
\hline 8.24 & 18.40 & 0.0692 & 33.8 & 67.6 & 2.545 & \\
\hline 6.06 & 18.40 & 0.0692 & 33.8 & 67.6 & 2.545 & \\
\hline 3.31 & 16.38 & 0.0692 & 33.8 & 67.6 & 2.545 & \\
\hline 8.02 & 19.31 & 0.0692 & 33.8 & 67.6 & 2.545 & \\
\hline 7.68 & 21.13 & 0.0692 & 33.8 & 67.6 & 2.545 & \\
\hline 4.99 & 25.22 & 0.0692 & 33.8 & 67.6 & 2.545 & \\
\hline 5.50 & 25.55 & 0.0692 & 33.8 & 67.6 & 2.545 & \\
\hline 8.24 & 27.30 & 0.0692 & 33.8 & 67.6 & 2.545 & \\
\hline 10.43 & 29.19 & 0.0692 & 33.8 & 67.6 & 2.545 & \\
\hline 11.10 & 28.99 & 0.0692 & 33.8 & 67.6 & 2.545 & \\
\hline 7.74 & 32.96 & 0.0692 & 33.8 & 67.6 & 2.545 & \\
\hline 6.90 & 35.95 & 0.0692 & 33.8 & 67.6 & 2.545 & \\
\hline 8.52 & 34.84 & 0.0692 & 33.8 & 67.6 & 2.545 & \\
\hline 9.03 & 34.84 & 0.0692 & 33.8 & 67.6 & 2.545 & \\
\hline 11.55 & 34.06 & 0.0692 & 33.8 & 67.6 & 2.545 & \\
\hline 12.22 & 38.29 & 0.0692 & 33.8 & 67.6 & 2.545 & \\
\hline 10.21 & 38.87 & 0.0692 & 33.8 & 67.6 & 2.545 & \\
\hline 9.36 & 38.29 & 0.0692 & 33.8 & 67.6 & 2.545 & \\
\hline 7.29 & 39.59 & 0.0692 & 33.8 & 67.6 & 2.545 & \\
\hline 7.74 & 43.94 & 0.0692 & 33.8 & 67.6 & 2.545 & \\
\hline 11.33 & 43.94 & 0.0692 & 33.8 & 67.6 & 2.545 & \\
\hline 10.93 & 45.37 & 0.0692 & 33.8 & 67.6 & 2.545 & \\
\hline 12.90 & 44.14 & 0.0692 & 33.8 & 67.6 & 2.545 & \\
\hline 8.30 & 48.56 & 0.0692 & 33.8 & 67.6 & 2.545 & \\
\hline 12.11 & 51.09 & 0.0692 & 33.8 & 67.6 & 2.545 & \\
\hline 14.30 & 49.66 & 0.0692 & 33.8 & 67.6 & 2.545 & \\
\hline 15.53 & 54.41 & 0.0692 & 33.8 & 67.6 & 2.545 & \\
\hline 13.79 & 55.06 & 0.0692 & 33.8 & 67.6 & 2.545 & \\
\hline 15.64 & 56.23 & 0.0692 & 33.8 & 67.6 & 2.545 & \\
\hline 15.00 & 20.00 & 0.0692 & 5 & 36 & 1.4 & Kato and Wen ${ }^{17}$; Material used: coal $\left(\rho=1.4 \mathrm{~g} / \mathrm{cm}^{3}\right)$ \\
\hline 24.00 & 40.00 & 0.0692 & 5 & 36 & 1.4 & \\
\hline 12.50 & 20.00 & 0.0692 & 5 & 24 & 1.4 & \\
\hline 21.00 & 40.00 & 0.0692 & 5 & 24 & 1.4 & \\
\hline 9.80 & 20.00 & 0.0692 & 5 & 18 & 1.4 & \\
\hline 16.00 & 40.00 & 0.0692 & 5 & 18 & 1.4 & \\
\hline 22.00 & 60.00 & 0.0692 & 5 & 18 & 1.4 & \\
\hline 27.00 & 80.00 & 0.0692 & 5 & 18 & 1.4 & \\
\hline 1.40 & 8.00 & 0.0117 & 1.7 & 2.4 & 2.61 & Darton et al. ${ }^{8} ;$ Material used: sand $\left(\rho=2.61 \mathrm{~g} / \mathrm{cm}^{3}\right)$ \\
\hline 1.70 & 18.00 & 0.0117 & 1.7 & 2.4 & 2.61 & \\
\hline 2.00 & 25.00 & 0.0117 & 1.7 & 2.4 & 2.61 & \\
\hline 2.20 & 32.00 & 0.0117 & 1.7 & 2.4 & 2.61 & \\
\hline 2.60 & 42.00 & 0.0117 & 1.7 & 2.4 & 2.61 & \\
\hline 3.20 & 50.00 & 0.0117 & 1.7 & 2.4 & 2.61 & \\
\hline 3.60 & 68.00 & 0.0117 & 1.7 & 2.4 & 2.61 & \\
\hline 2.20 & 8.00 & 0.0117 & 1.7 & 5.2 & 2.61 & \\
\hline 3.00 & 20.00 & 0.0117 & 1.7 & 5.2 & 2.61 & \\
\hline 3.50 & 25.00 & 0.0117 & 1.7 & 5.2 & 2.61 & \\
\hline 4.30 & 33.00 & 0.0117 & 1.7 & 5.2 & 2.61 & \\
\hline
\end{tabular}


RESEARCH ARTICLES

Table 2. (Contd)

\begin{tabular}{|c|c|c|c|c|c|c|}
\hline$D_{\mathrm{b}}(\mathrm{cm})$ & $h(\mathrm{~cm})$ & $d_{\mathrm{p}}(\mathrm{cm})$ & $U_{\mathrm{mf}}(\mathrm{cm} / \mathrm{s})$ & $U(\mathrm{~cm} / \mathrm{s})$ & $\rho\left(\mathrm{g} / \mathrm{cm}^{3}\right)$ & Authors \\
\hline 4.40 & 43.00 & 0.0117 & 1.7 & 5.2 & 2.61 & \\
\hline 6.00 & 51.00 & 0.0117 & 1.7 & 5.2 & 2.61 & \\
\hline 7.80 & 68.00 & 0.0117 & 1.7 & 5.2 & 2.61 & \\
\hline 3.00 & 9.00 & 0.0117 & 1.7 & 7.5 & 2.61 & \\
\hline 4.50 & 18.00 & 0.0117 & 1.7 & 7.5 & 2.61 & \\
\hline 8.20 & 42.00 & 0.0117 & 1.7 & 7.5 & 2.61 & \\
\hline 9.80 & 52.00 & 0.0117 & 1.7 & 7.5 & 2.61 & \\
\hline 0.50 & 0.50 & 0.0083 & 1.8 & 9 & 2.66 & Werther': Material used: quartz sand $\left(\rho=2.66 \mathrm{~g} / \mathrm{cm}^{3}\right)$ \\
\hline 1.00 & 1.00 & 0.0083 & 1.8 & 9 & 2.66 & \\
\hline 1.20 & 1.20 & 0.0083 & 1.8 & 9 & 2.66 & \\
\hline 2.80 & 10.00 & 0.0083 & 1.8 & 9 & 2.66 & \\
\hline 3.60 & 15.00 & 0.0083 & 1.8 & 9 & 2.66 & \\
\hline 4.40 & 20.00 & 0.0083 & 1.8 & 9 & 2.66 & \\
\hline 5.00 & 25.00 & 0.0083 & 1.8 & 9 & 2.66 & \\
\hline 6.30 & 30.00 & 0.0083 & 1.8 & 9 & 2.66 & \\
\hline 0.70 & 0.40 & 0.0083 & 1.8 & 9 & 2.66 & \\
\hline 1.30 & 1.50 & 0.0083 & 1.8 & 9 & 2.66 & \\
\hline 1.50 & 2.00 & 0.0083 & 1.8 & 9 & 2.66 & \\
\hline 1.80 & 3.00 & 0.0083 & 1.8 & 9 & 2.66 & \\
\hline 2.00 & 4.50 & 0.0083 & 1.8 & 9 & 2.66 & \\
\hline 4.28 & 14 & 0.0275 & 6.5 & 97 & 2.5 & \\
\hline 5.12 & 19 & 0.0275 & 6.5 & 97 & 2.5 & \\
\hline 5.68 & 28 & 0.0275 & 6.5 & 97 & 2.5 & \\
\hline 5.88 & 31 & 0.0275 & 6.5 & 97 & 2.5 & \\
\hline 2.58 & 5 & 0.039 & 12 & 180 & 2.5 & \\
\hline 3.8 & 6 & 0.039 & 12 & 180 & 2.5 & \\
\hline 4.48 & 8 & 0.039 & 12 & 180 & 2.5 & \\
\hline 5.87 & 16 & 0.039 & 12 & 180 & 2.5 & \\
\hline 5.6 & 22 & 0.039 & 12 & 180 & 2.5 & \\
\hline 5.43 & 26 & 0.039 & 12 & 180 & 2.5 & \\
\hline 5.73 & 30 & 0.039 & 12 & 180 & 2.5 & \\
\hline 5.62 & 34 & 0.039 & 12 & 180 & 2.5 & \\
\hline 4.58 & 4.5 & 0.0462 & 24.5 & 367.5 & 2.5 & \\
\hline 5.35 & 6.5 & 0.0462 & 24.5 & 367.5 & 2.5 & \\
\hline 6.54 & 10 & 0.0462 & 24.5 & 367.5 & 2.5 & \\
\hline 6.09 & 12.5 & 0.0462 & 24.5 & 367.5 & 2.5 & \\
\hline 8.72 & 17.5 & 0.0462 & 24.5 & 367.5 & 2.5 & \\
\hline 8.97 & 22 & 0.0462 & 24.5 & 367.5 & 2.5 & \\
\hline 9.36 & 28 & 0.0462 & 24.5 & 367.5 & 2.5 & \\
\hline 9.66 & 34 & 0.0462 & 24.5 & 367.5 & 2.5 & \\
\hline 7.144 & 5 & 0.138 & 77 & 1155 & 2.5 & \\
\hline
\end{tabular}


Steps (ii) to (iv) are performed iteratively (see the flowchart in Figure 2) until a best-fitting candidate solution (expression) is secured. An in-depth treatment of the GPSR procedure can be found in several studies ${ }^{24-27}$.

\section{Results and discussion}

Five parameters, namely particle diameter $\left(d_{\mathrm{p}}\right)$, height of bubble in fluidized bed $(h)$, input velocity $(U)$, density of particles $(\rho)$ and minimum fluidization velocity $\left(U_{\mathrm{mf}}\right)$, were used as inputs for obtaining the GP-based equation predicting the bubble diameter $\left(D_{\mathrm{b}}\right)$. Data pertaining to experiments listed in Table 2 were used as the example data set for developing this model. The data set was randomly partitioned into training, test and validation sets containing data of 86,25 and 12 experiments respectively. While the first set was used in constructing the model, the test set and validation set were used in assessing the generalization performance of the developed model.

The GP-based equation predicting the $D_{\mathrm{b}}$ magnitude was developed using Eureqa Formulize (EF) software package $^{28}$. This package has been optimized for producing parsimonious (i.e. with reduced complexity) models possessing good generalization ability. Towards securing an optimal GP-based model having an excellent $D_{\mathrm{b}}$ prediction and generalization performance, the effects of GP procedural parameters such as the size of training and test sets as also the various input normalization schemes, were rigorously examined. The prediction accuracy and

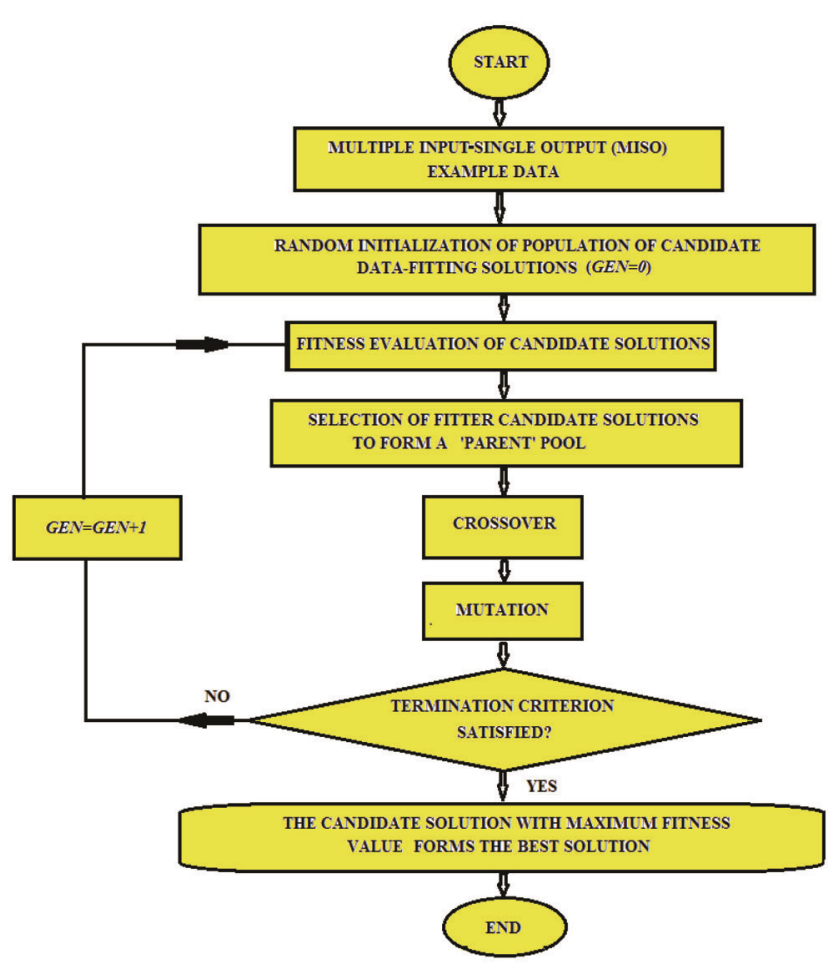

Figure 2. Flowchart of a generic GP implementation. generalization performance of the GP-based model were evaluated by computing the coefficient of correlation (CC), root mean square error (RMSE), and mean absolute per cent error (MAPE) values using the experimental (target) and the corresponding model-predicted values of

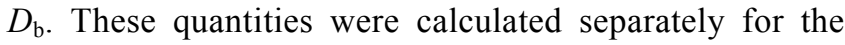
training, test and validation data sets. The overall best GP-based model was selected on the basis of its high CC and low RMSE and MAPE magnitudes pertaining to both training and test data sets. The optimal GP-based equation for the prediction of the bubble diameter is as follows

$$
\begin{array}{r}
D_{\mathrm{b}}=2.1299+74.82 d_{\mathrm{p}}+0.1817 h \\
+0.154\left(U / U_{\mathrm{mf}}\right)-1.848 \rho .
\end{array}
$$

Table 3 lists the values of CC, RMSE and MAPE with respect to the bubble diameter predictions made by the GP-based eq. (1) and their corresponding experimental magnitudes in the training, test, and validation sets. From the high $(\approx 0.83)$ and comparable magnitudes of $\mathrm{CC}$ as also low and comparable magnitudes of RMSEs $(\approx 0.018)$ and MAPES $(\approx 0.23)$ pertaining to the training, test and validation data listed in Table 3 , it is clear that the GPbased model possesses good $D_{\mathrm{b}}$ prediction accuracy and generalization capability.

The GP-based equation was used for calculating the bubble diameters of particles of different sizes and the values obtained thereby have been compared with the corresponding experimental $D_{\mathrm{b}}$ values as also the predictions made by the existing bubble diameter equations; this comparison is shown in Figures 3 to 7 . In these plots, the experimental $D_{\mathrm{b}}$ values (see Table 2) are compared with those computed using the equations from other studies $^{8-13,15}$ and the GP-based eq. (1). Figure 3 shows a comparison of the predictions of the bubble diameter with respect to the coal particles of $692 \mu \mathrm{m}$ diameter and density equal to $1.4 \mathrm{gm} / \mathrm{cm}^{3}$ (ref. 17). Here, it is seen that all the currently available equations and the GP based eq. (1) underpredict the experimentally observed bubble diameter. In the initial stage of bubble travel, the prediction of the GP-based equation is closest to the experimental $D_{\mathrm{b}}$ values when compared to the predictions of other equations. Figure 4 provides a comparison of the $D_{\mathrm{b}}$ predictions corresponding to the glass particles with $d_{\mathrm{p}}$ and $\rho$ equal to $692 \mu \mathrm{m}$ and $2.54 \mathrm{~g} / \mathrm{cm}^{3}$ respectively ${ }^{22}$. These experimental $D_{\mathrm{b}}$ data have been predicted well by the

Table 3. Prediction and generalization performance of the GP-based model

\begin{tabular}{lccc}
\hline & Training set data & Test set data & Validation set data \\
\hline CC & 0.889 & 0.755 & 0.846 \\
RMSE & 0.026 & 0.012 & 0.016 \\
MAPE & 0.361 & 0.202 & 0.143 \\
\hline
\end{tabular}




\section{RESEARCH ARTICLES}

GP-based equation and those proposed by Rowe ${ }^{15}$, Hilligardt and Werther ${ }^{9}$ and Yasui ${ }^{11}$. Figure 5 exhibits a comparison of the $D_{\mathrm{b}}$ predictions made by various equations pertaining to the experimental data presented by Darton et $a l .{ }^{8}$ using sand particles of diameter and density respectively, equal to $117 \mu \mathrm{m}$ and $2.61 \mathrm{~g} / \mathrm{cm}^{3}$. The bubble diameter magnitudes shown in Figure $5 a$ correspond to the injection velocity of $5.3 \mathrm{~cm} / \mathrm{s}$ while it is $7.5 \mathrm{~cm} / \mathrm{s}$ for Figure $5 b$. In Figure $5 a$, it is seen that the equation by Werther ${ }^{9}$ predicts the experimental $D_{\mathrm{b}}$ values with maximum accuracy and the GP-based model seems to overpredict the bubble diameter in the last stage of the bubble travel. While all other equations underpredict the experimental bubble diameter magnitudes in Figure $5 \mathrm{~b}$, these are predicted with an excellent accuracy by the GP-based model and the equations of Werther ${ }^{9}$ and Lim et al. ${ }^{10}$. Figure 6 shows the bubble diameter predictions for the

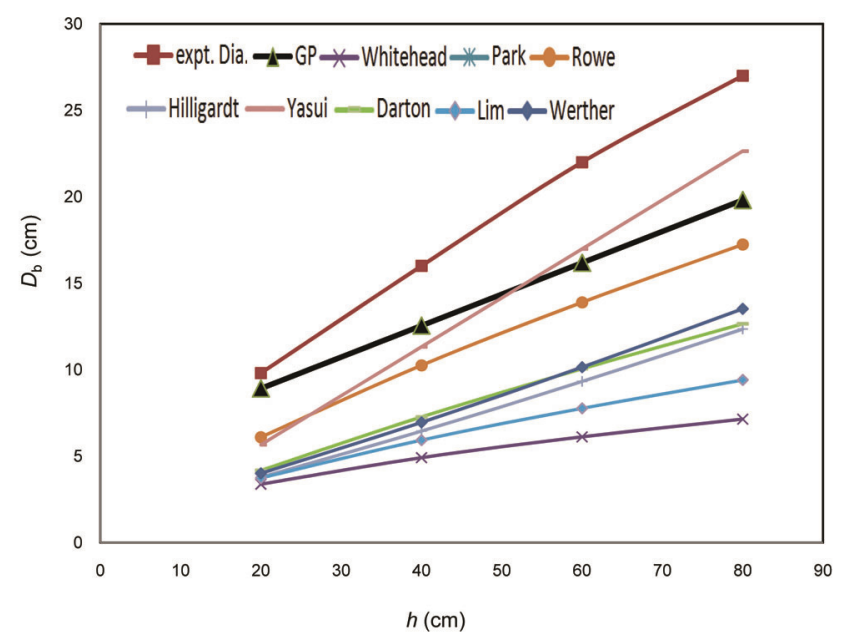

Figure 3. A comparison of the experimentally determined bubble diameter values of the fluidized coal particles with those predicted by a number of existing models and the proposed GP-based equation.

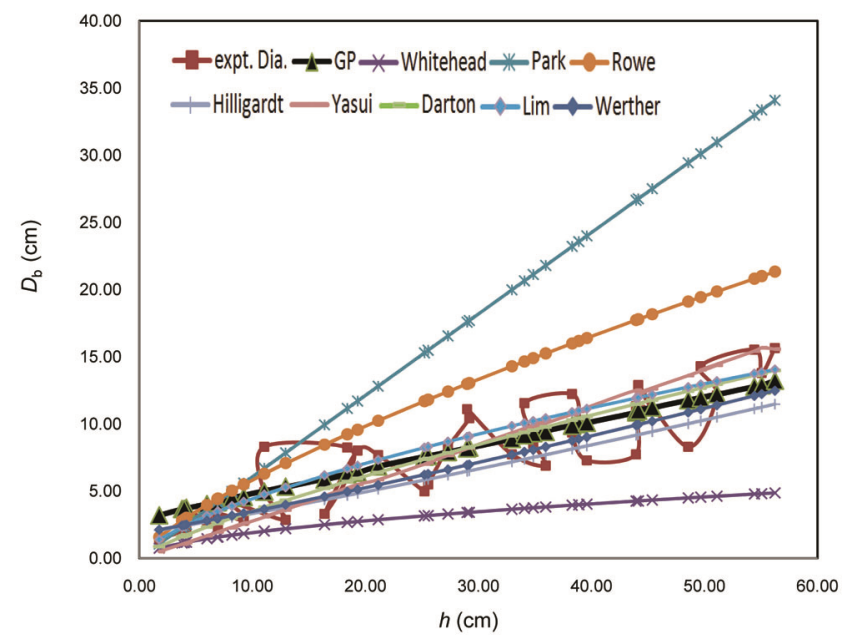

Figure 4. A comparison of the experimentally determined bubble diameter values of the fluidized glass particles with those predicted by a number of existing models and the proposed GP-based equation. experimental data provided by Werther $^{9}$ using quartz sand. Panels $a$ and $b$ of this figure correspond to the data obtained from two different reactors of diameters $20 \mathrm{~cm}$ and $100 \mathrm{~cm}$ respectively. In both these figures, the experimental bubble diameter magnitudes have been predicted with an excellent accuracy by the GP-based equation and Werther ${ }^{9}$ equations. Figure $7 a-d$ shows a comparison of the experimental and model-predicted $D_{\mathrm{b}}$ values pertaining to the glass particles of sizes 275, 390, 462 and $1380 \mu \mathrm{m}$ respectively. Here, it is observed that the equation by Whitehead and Young ${ }^{12}$ predicted the experimental $D_{\mathrm{b}}$ values reasonably accurately for the particle diameter magnitudes of 275, 390 and $462 \mu \mathrm{m}$, whereas the predictions of the model by Lim et al. ${ }^{10}$ closely resemble the experimentally monitored bubble diameters for the particle diameter of $1380 \mu \mathrm{m}$. Notably, for all the four particle sizes, the GP-based equation closely predicts the experimentally observed bubble diameter values.

Table 4 shows a comparison of the CC, RMSE and MAPE values pertaining to experimental $D_{\mathrm{b}}$ values and
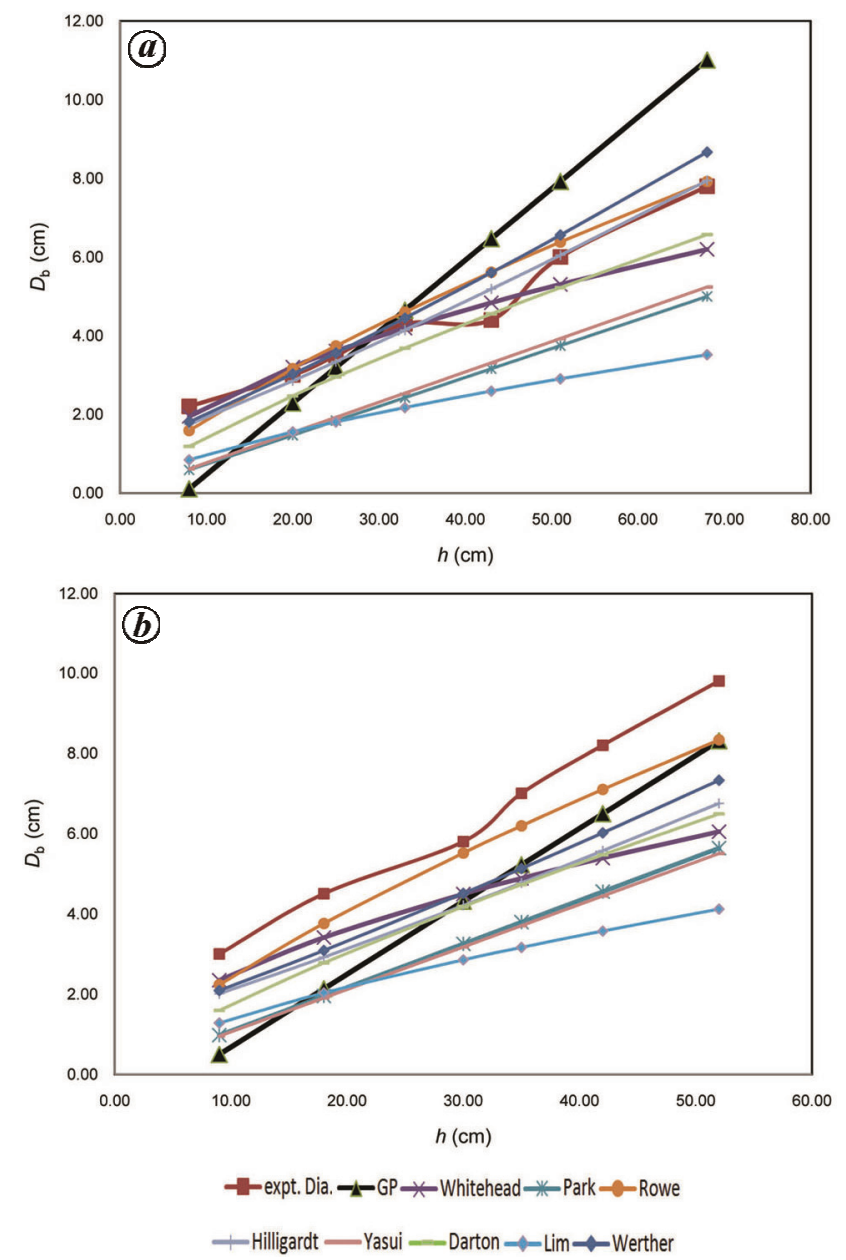

Figure 5. A comparison of the experimentally determined bubble diameter values of the fluidized sand particles with those predicted by a number of existing models and the proposed GP-based equation; $\boldsymbol{a}, \boldsymbol{b}$, Correspond to the injection velocities of 5.3 and $7.5 \mathrm{~cm} / \mathrm{s}$ respectively. 

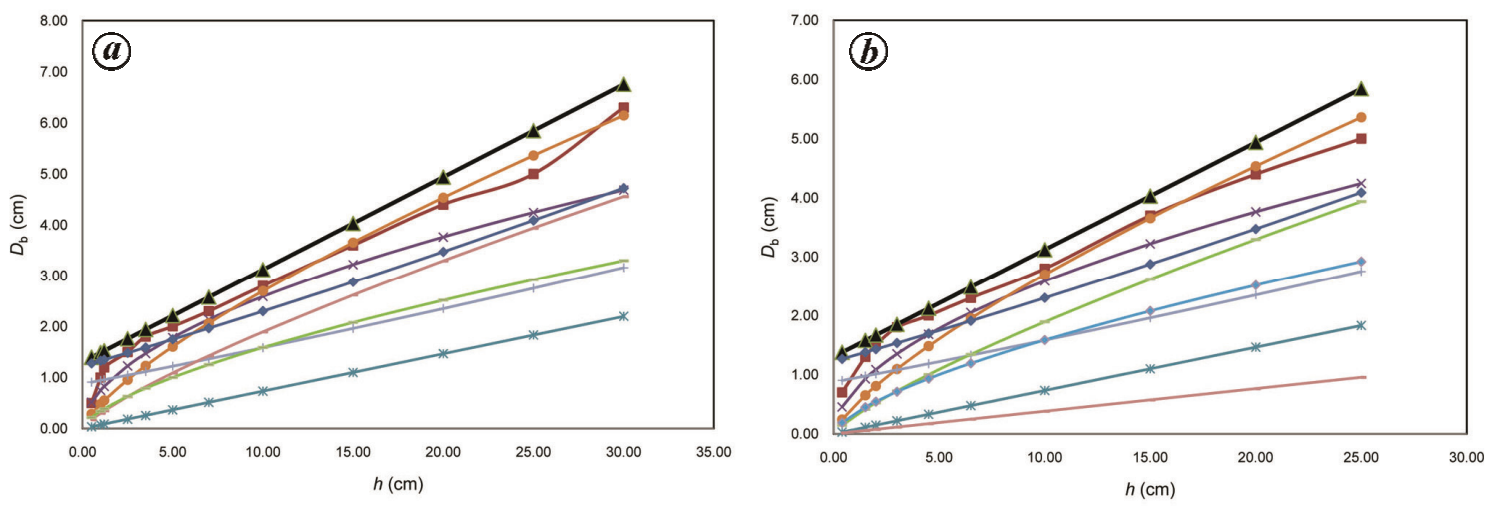

$\rightarrow$-expt. Dia $-\mathbf{L}-\mathrm{GP} *$ Whitehead $*$ Park $\rightarrow$-Rowe + Hilligardt — Yasui —Darton $\rightarrow-$ Lim $\multimap-$ Werther

Figure 6. A comparison of the experimentally determined bubble diameter values of the fluidized quartz sand particles with those predicted by a number of existing models and the proposed GP-based equation; $\boldsymbol{a}, \boldsymbol{b}$, correspond to the experimental data obtained from columns of diameters 20 and $100 \mathrm{~cm}$ respectively.
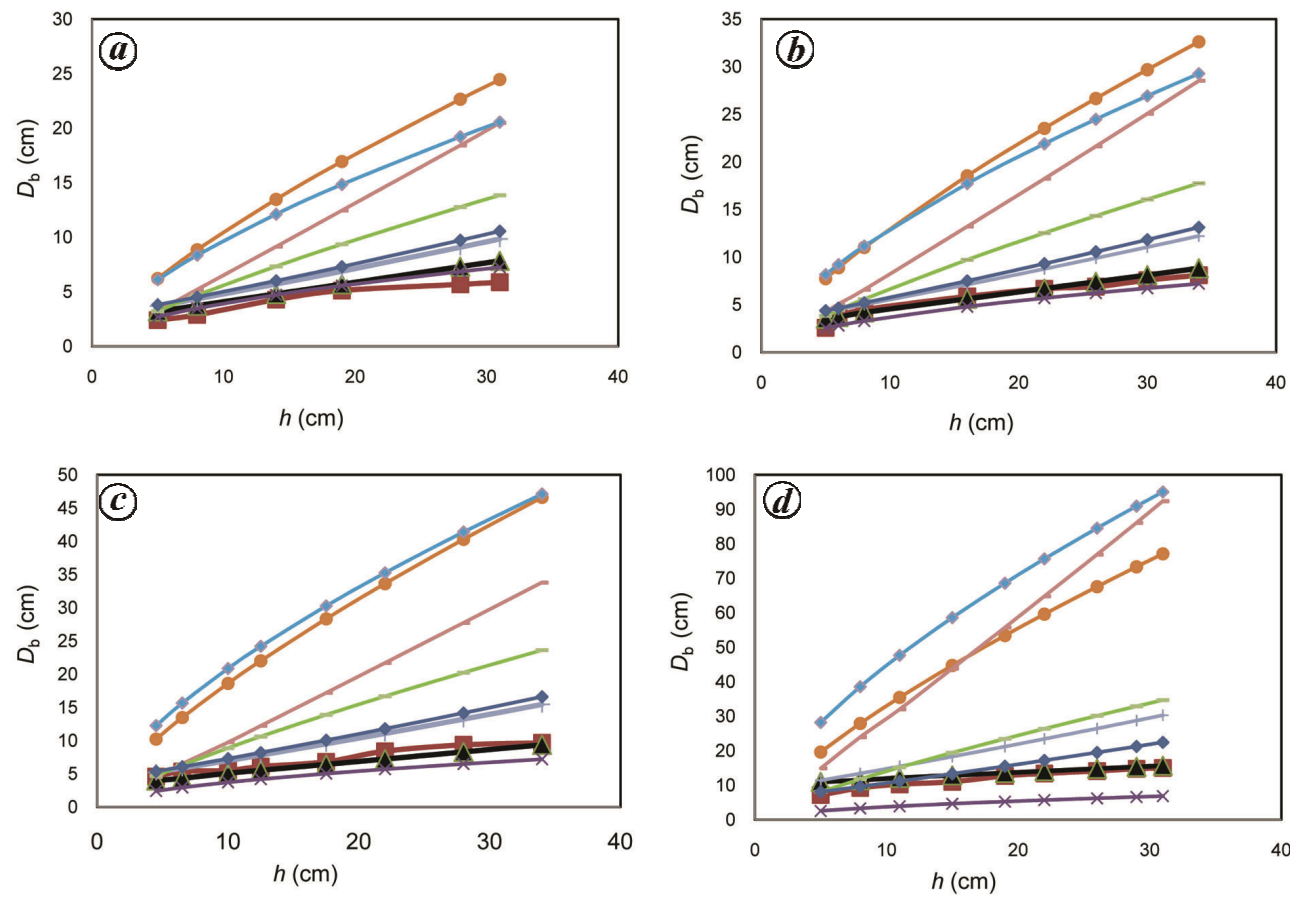

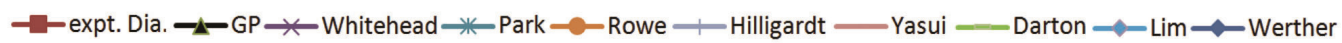

Figure 7. Comparison of the experimentally determined bubble diameter values of the fluidized glass particles, with the corresponding predictions made by a number of existing models and the proposed GP-based equation for the particle diameters of $275 \mu \mathrm{m}(\boldsymbol{a}), 390 \mu \mathrm{m}(\boldsymbol{b}), 462 \mu \mathrm{m}(\boldsymbol{c})$, and $1380 \mu \mathrm{m}(\boldsymbol{d})$.

the corresponding predictions made by the GP-based model and also the equations proposed by other studies ${ }^{7-13,15}$. These quantities were evaluated using the entire data set listed in Table 2. It is clearly seen in Table 4 that there exists a significant variation in $D_{\mathrm{b}}$ prediction accuracies possessed by the currently available models. Among the existing models, the one proposed by Werther ${ }^{9}$ exhibited the highest prediction accuracy $(\mathrm{CC}=0.6999$, $\mathrm{RMSE}=$ 0.0369 , MAPE $=0.3237)$. In comparison, the GP-based equation predicted the bubble diameter values with a significantly higher accuracy $(\mathrm{CC}=0.8864, \mathrm{RMSE}=0.0021$,
MAPE $=0.3092$ ). The extent of improvement in the $D_{\mathrm{b}}$ prediction accuracy exhibited by the GP-based model over the best performing existing model by Werther ${ }^{9}$ is $\approx 26 \%$.

\section{Conclusion}

Using extensive experimental data involving a fluidized bed, it has been shown in this study that a number of currently available models for the prediction of bubble diameter $\left(D_{\mathrm{b}}\right)$ yield suboptimal predictions. Specifically, 
Table 4. A comparison of the $D_{\mathrm{b}}$ prediction performance of the GP-based and currently available four equations

\begin{tabular}{|c|c|c|c|c|c|c|c|c|c|}
\hline & \multicolumn{9}{|c|}{$D_{\mathrm{b}}$ prediction equations } \\
\hline & $\begin{array}{l}\text { GP-based } \\
\text { (eq. 1) }\end{array}$ & $\begin{array}{l}\text { Yasui and } \\
\text { Johanson }^{11}\end{array}$ & $\begin{array}{c}\text { Whitehead and } \\
\text { Young }^{12}\end{array}$ & Park et al. ${ }^{13}$ & Werther ${ }^{9}$ & $\begin{array}{l}\text { Hilligart and } \\
\text { Rowe } \text { et } a l .{ }^{15}\end{array}$ & Werther ${ }^{7}$ & Darton et al. ${ }^{8}$ & Lim et al. ${ }^{10}$ \\
\hline $\mathrm{CC}$ & 0.886 & 0.545 & 0.643 & 0.524 & 0.699 & 0.538 & 0.631 & 0.632 & 0.429 \\
\hline RMSE & 0.002 & 0.142 & 0.052 & 0.718 & 0.036 & 0.141 & 0.045 & 0.053 & 0.174 \\
\hline MAPE & 0.309 & 0.864 & 0.391 & 1.900 & 0.323 & 0.983 & 0.371 & 0.450 & 1.072 \\
\hline
\end{tabular}

these equations exhibit a large variation in predicting the magnitudes of the bubble diameter over its wide range. Accordingly, a new exclusively data-driven model has been developed for computing the bubble diameter values in fluidized beds using a computational intelligence formalism, namely GP. The performance of the proposed GP-based model has been compared rigorously with the experimental data and that of several currently available $D_{\mathrm{b}}$ prediction models. This comparison shows a good performance by the GP-based model over a wide $D_{\mathrm{b}}$ range. Owing to its superior $D_{\mathrm{b}}$ prediction accuracy, the GPbased model proposed in this study can be gainfully used in the design of fluidized beds using various types of particles.

1. Kwauk, M. and Li, H. Z., Handbook of Fluidization, Chemical Industry Press, Beijing (in Chinese), 2007.

2. Harrison, D. and Leung, L. S., Bubble formation at an orifice in a fluidized bed. Trans. Inst. Chem. Eng., 1961, 34, 409-414.

3. Zenz, F. A., Bubble formation and grid design. Inst. Chem. Eng. Symp. Ser., 1968, 30, 136-139.

4. Nieuwland, J. J., Hydrodynamic modeling of gas-solid two phase flows. Ph D thesis, Twenty University, 1995.

5. Yang, Y. M. and Maa, J. R., Bubble coalescence in dilute surfactant solutions. J. Colloid Interf. Sci., 1984, 98, 120-125.

6. Caram, H. S. and Hsu, K. K., Bubble formation and gas leakage in fluidized beds. Chem. Eng. Sci., 1986, 41, 1445-1453.

7. Hilligardt, K. and Werther, J., Local bubble gas hold up and expansion of gas-solid fluidized beds. German Chem. Eng., 1986, 9, 215-221.

8. Darton, R. C., La Nauze, R. D., Davidson, J. F. and Harrison, D., Bubble growth due to coalescence in fluidized beds. Trans. Inst. Chem. Eng., 1977, 55, 274-280.

9. Werther, J., The influence of the bed diameter on the hydrodynamics of gas fluidized beds. AIChE Meeting Detroit, 1973.

10. Lim. K. S., Gururaja, V. S. and Agrawal, P. K., Mixing and homogenous solids in bubbling fluidized beds: theoretical modeling and experimental investigation using digital image analysis. Chem. Eng. Sci., 1993, 48, 2251-2265.

11. Yasui, G. and Johanson, L. N., Characteristics of gas pockets in fluidized beds. AIChE J., 1958, 4, 445.

12. Whitehead, A. B. and Young, A. D., Fluidization performance in large scale equipment Part-I. In Proceedings of International Symposium on Fluidization, Eindhoven, Netherlands, 1967, p. 84.

13. Park, W. H., Kang, W. K., Copes, C. E. and Osberg, G. L., The properties of bubbles in fluidized beds of conducting particles as measured by an electro resistivity probe. Chem. Eng. Sci., 1969, 24, 851 .

14. Geldart, D., the size and frequency if bubbles in two and three dimensional fluidized beds. Powder Technol., 1970, 4, 41.

15. Rowe, P. N., Prediction of bubble size in gas fluidized bed. Chem. Eng. Sci., 1976, 31, 285-288.
16. Mori, S. and Wen, C. Y., Estimation of bubble diameter in gaseous fluidized beds. AIChE J., 1975, 21, 109-115.

17. Kato, K. and Wen, C. Y., Bubble assemblage, model for fluidized bed catalytic reactors. Chem. Eng. Sci., 1969, 24, 1351-1369.

18. Shen, L., Johnsson, F. and Leckner, B., Digital image analysis of hydrodynamics two dimensional bubbling fluidized beds. Chem. Eng. Sci., 2004, 59, 2601-2617.

19. Horio, M. and Nonaka, A., A generalized bubble diameter correlation for gas solid fluidized beds. AIChE J., 1987, 33, 18651872.

20. Toor, F. D. and Calderbank, P. H., Reaction kinetics in gasfluidized catalyst beds; Part II: Mathematical models. In Proceedings International Symposium on Fluidization, Netherlands University Press, Amsterdam, 1967, pp. 373-392.

21. Peters, M. H., Fan, L. S. and Sweeney, T. L., Reactant dynamics in catalytic fluidized bed reactors with flow reversal of gas in emulsion phase. Chem. Eng. Sci., 1982, 37, 553-565.

22. Patil, D. J., van Sint Annaland, M. and Kuipers, J. A. M., Critical comparison of hydrodynamic models for gas-solid fluidized beds - Part II: freely bubbling gas-solid fluidized beds. Chem. Eng. Sci., 2005, 60, 73-84.

23. Farshi, A., Javaherizadeh, H. and Hamzavi-Abedi, M. A., An investigation of the effect of bubble diameter on the performance of gas solid fluidized bed reactor and two phase modeling of bubbling fluidized bed reactor in melamine production. Pet. Coal, 2008, 50(1), 11-22.

24. Koza, J. R., Genetically breeding populations of computer programs to solve problems in artificial intelligence. In Proceedings of the 2nd International IEEE Conference on Tools for Artificial Intelligence, 6-9 November 1990, pp. 819-827; http://dx.doi.org/ 10.1109/TAI.1990.130444.

25. Poli, R., Langdon, W. and Mcphee, N., A field guide to genetic programming, 2008; http://lulu.com and freely available at http://www.gp-field-guide.org.uk

26. Goel, P., Bapat, S., Vyas, R., Tambe, A. and Tambe, S. S., Genetic programming based quantitative structure - retention relationships for the prediction of kovats retention indices. J. Chromatogr. A., 2015, 1420, 98-109; doi:10.1016/j.chroma.2015.09.086.

27. Shrinivas, K., Kulkarni, R., Saif Shaikh, Ghorpade, R., Renu Vyas, Tambe, S. S., Ponrathnam, S. and Kulkarni, B. D., Prediction of reactivity ratios in free radical copolymerization from monomer resonance-polarity (Q-e) parameters: genetic programming-based models. Int. J. Chem. React. Eng., Published online on 4 March 2015, doi 10.1515/ijcre-2014-0039.

28. Schmidt, M. and Lipson, H., Distilling free-form natural laws from experimental data. Science, 2009, 324, 81-85.

ACKNOWLEDGEMENT. S.S.T. acknowledges a partial support for this study by the Council of Scientific and Industrial Research, Government of India, New Delhi, under TAPCOAL Network Project.

Received 2 March 2016; accepted 17 May 2016

doi: $10.18520 / \mathrm{cs} / \mathrm{v} 115 / \mathrm{i} 10 / 1904-1912$ 